Supplement of J. Bone Joint Infect., 6, 135-140, 2021

https://doi.org/10.5194/jbji-6-135-2021-supplement

(c) Author(s) 2021. CC BY 4.0 License.

(c) (1)

Supplement of

\title{
Prospective evaluation of pin site infections in 39 patients treated with external ring fixation
}

Mats Bue et al.

Correspondence to: Juozas Petruskevicius (juopet@rm.dk)

The copyright of individual parts of the supplement might differ from the article licence. 


\section{Supplement}

Pin site registration sheet.

\section{Table S1:}

Name of the patient + ID:

10 Date of primary ring fixation (dd-mm-yy):

Follow-up

\begin{tabular}{|c|l|c|c|}
\hline & $\begin{array}{l}\text { Date } \\
\mathrm{dd} / \mathrm{mm}-\mathrm{yy}\end{array}$ & $\begin{array}{c}\text { Antibiotics since last } \\
\text { visit (Yes/No) }\end{array}$ & $\begin{array}{c}\text { Comments } \\
\text { (Pin care frequency,Antibiotics, dosage, hospitalisation, } \\
\text { other events) }\end{array}$ \\
\hline 1 & & & \\
\hline 2 & & & \\
\hline
\end{tabular}

Name of the patient + ID:

\section{Operations}

(primary ring fixation, re-operations, wire/pin removal, removal of rings, etc)

\begin{tabular}{|l|l|l|}
\hline & Date & \\
\hline 1 & & \\
& & \\
\hline 2 & & \\
\hline
\end{tabular}

\section{Figure S1:}

Draw the frame on the actual limb and side.

\section{Anatomical location}

Definition of the rings:

Distal diaphysis, (F-DD) Distal metaphysis (F-DM)

Tibia: $\quad$ Proximal metaphysis (PM)

Distal metaphysis (DM)

Foot: $\quad$ Foot frame

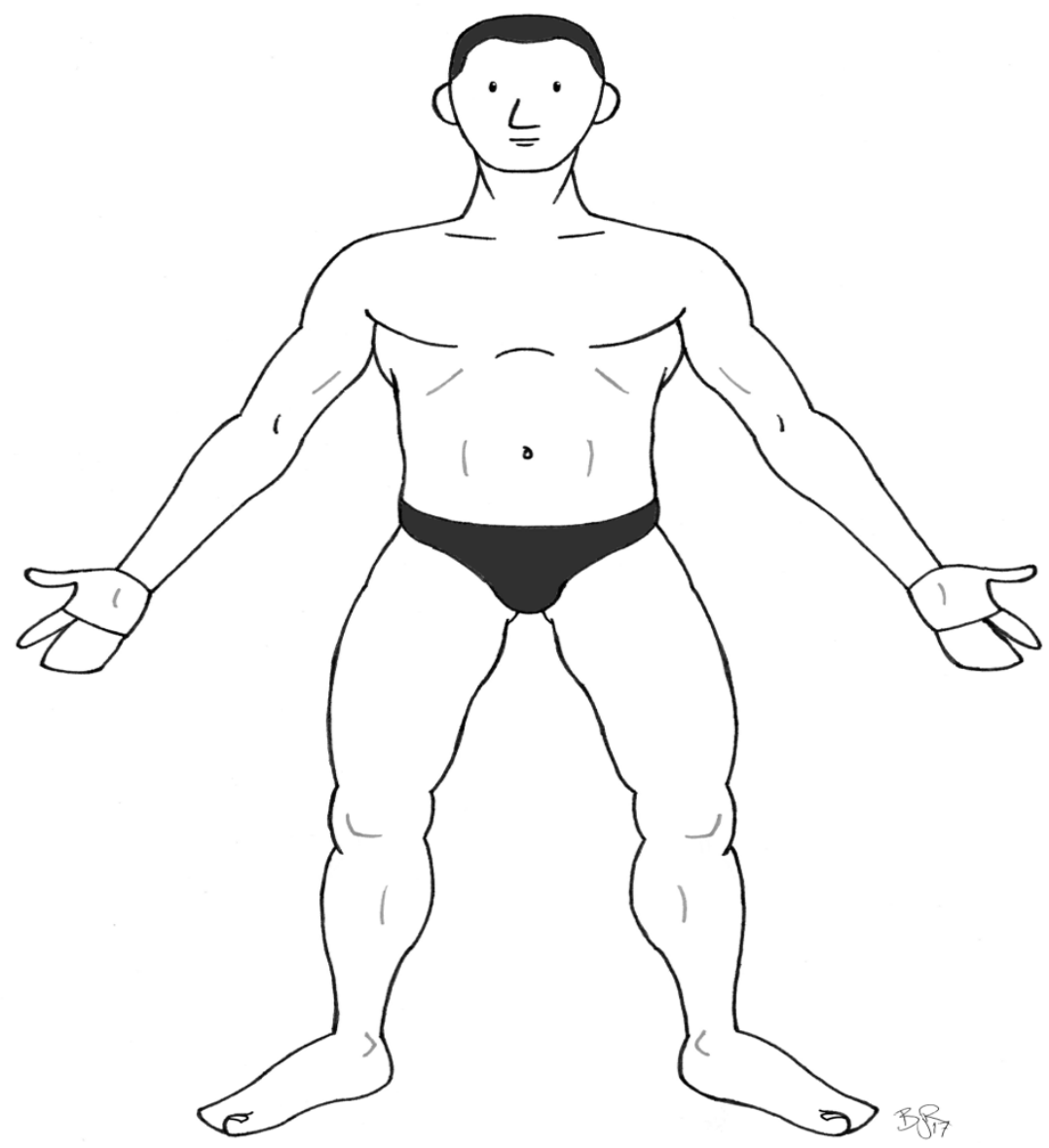


Table S2:

\section{Halfpins (HP) and Wires}

- Numbering of the pin sites starts from the most posterior-medial site and continues anti-clockwise on the right extremity (clockwise on left extremity): HP1, HP2 for halfpins, 1M, 2M for wires and so on)

- A wire always has two pin sites: medial and lateral (ex: $2 \mathrm{M}$ and $2 \mathrm{~L}$ ).

55 Registration of pin site infection

Note the grade of infection (CO 0-6, see at bottom of this sheet) in the empty cells

\begin{tabular}{|l|l|l|l|l|l|l|l|l|l|l|}
\hline Date & \multicolumn{7}{|c|}{ Tibia - Prox metaphysis } & \multicolumn{2}{c|}{ Comments } \\
\hline & $1 \mathrm{M}$ & $2 \mathrm{M}$ & $3 \mathrm{M}$ & $4 \mathrm{M}$ & $1 \mathrm{~L}$ & $2 \mathrm{~L}$ & $3 \mathrm{~L}$ & $4 \mathrm{~L}$ & & \\
\hline & & & & & & & & & & \\
\hline & & & & & & & & & & \\
\hline
\end{tabular}

\begin{tabular}{|l|l|l|l|l|l|}
\hline Date & \multicolumn{3}{|c|}{ Tibia - Prox diaphysis } & Comments \\
\hline & HP1 & HP2 & & & \\
\hline & & & & & \\
\hline & & & & & \\
\hline
\end{tabular}

60

\begin{tabular}{|l|l|l|l|l|l|}
\hline Date & \multicolumn{3}{|c|}{ Tibia - Distal diaphysis } & Comments \\
\hline & HP1 & HP2 & & & \\
\hline & & & & & \\
\hline & & & & & \\
\hline
\end{tabular}

\begin{tabular}{|l|l|l|l|l|l|l|l|l|l|l|}
\hline Date & \multicolumn{9}{|c|}{ Tibia - Distal metaphysis } & \multicolumn{2}{c|}{ Comments } \\
\hline & $1 \mathrm{M}$ & $2 \mathrm{M}$ & $3 \mathrm{M}$ & $4 \mathrm{M}$ & $1 \mathrm{~L}$ & $2 \mathrm{~L}$ & $3 \mathrm{~L}$ & $4 \mathrm{~L}$ & & \\
\hline & & & & & & & & & & \\
\hline & & & & & & & & & & \\
\hline
\end{tabular}

65

\begin{tabular}{|l|l|l|l|l|l|l|}
\hline Date & \multicolumn{5}{|c|}{ Femur - Distal diaphysis } & Comments \\
\hline & HP1 & HP2 & $1 \mathrm{M}$ & $1 \mathrm{~L}$ & & \\
\hline & & & & & & \\
\hline & & & & & & \\
\hline
\end{tabular}

\begin{tabular}{|l|l|l|l|l|l|l|}
\hline Date & \multicolumn{5}{|c|}{ Femur - Distal metaphysis } & \multicolumn{2}{c|}{ Comments } \\
\hline & HP1 & HP2 & $1 \mathrm{M}$ & $1 \mathrm{~L}$ & & \\
\hline & & & & & & \\
\hline & & & & & & \\
\hline
\end{tabular}

\begin{tabular}{|l|l|l|l|l|l|l|l|l|}
\hline Date & \multicolumn{7}{|c|}{ Footframe } & \multicolumn{2}{c|}{ Comments } \\
\hline & $1 \mathrm{M}$ & $2 \mathrm{M}$ & $3 \mathrm{M}$ & $3 \mathrm{~L}$ & $1 \mathrm{~L}$ & $2 \mathrm{~L}$ & & \\
\hline & & & & & & & & \\
\hline & & & & & & & & \\
\hline
\end{tabular}

70 
Figure 2S:

Femur: distal diaphysis or distal metaphysis with wires and half pins

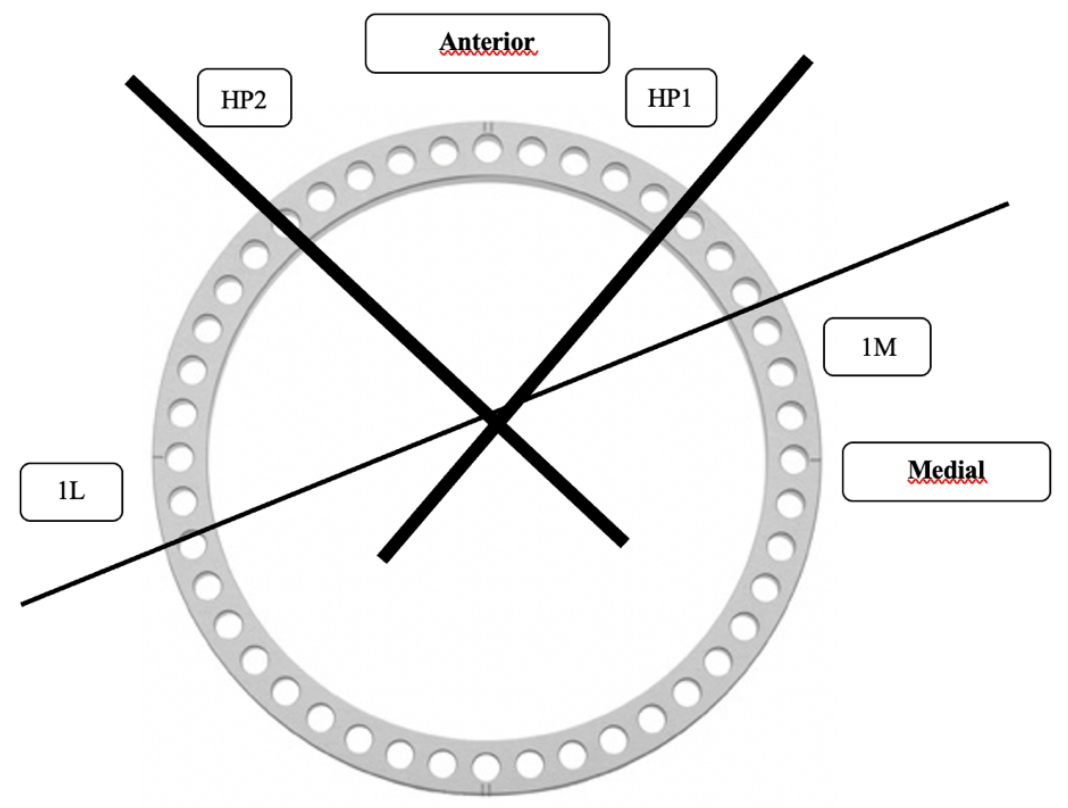

85 Figure 3S:

Tibia: proximal/distal metaphysis with wires (standard placement)

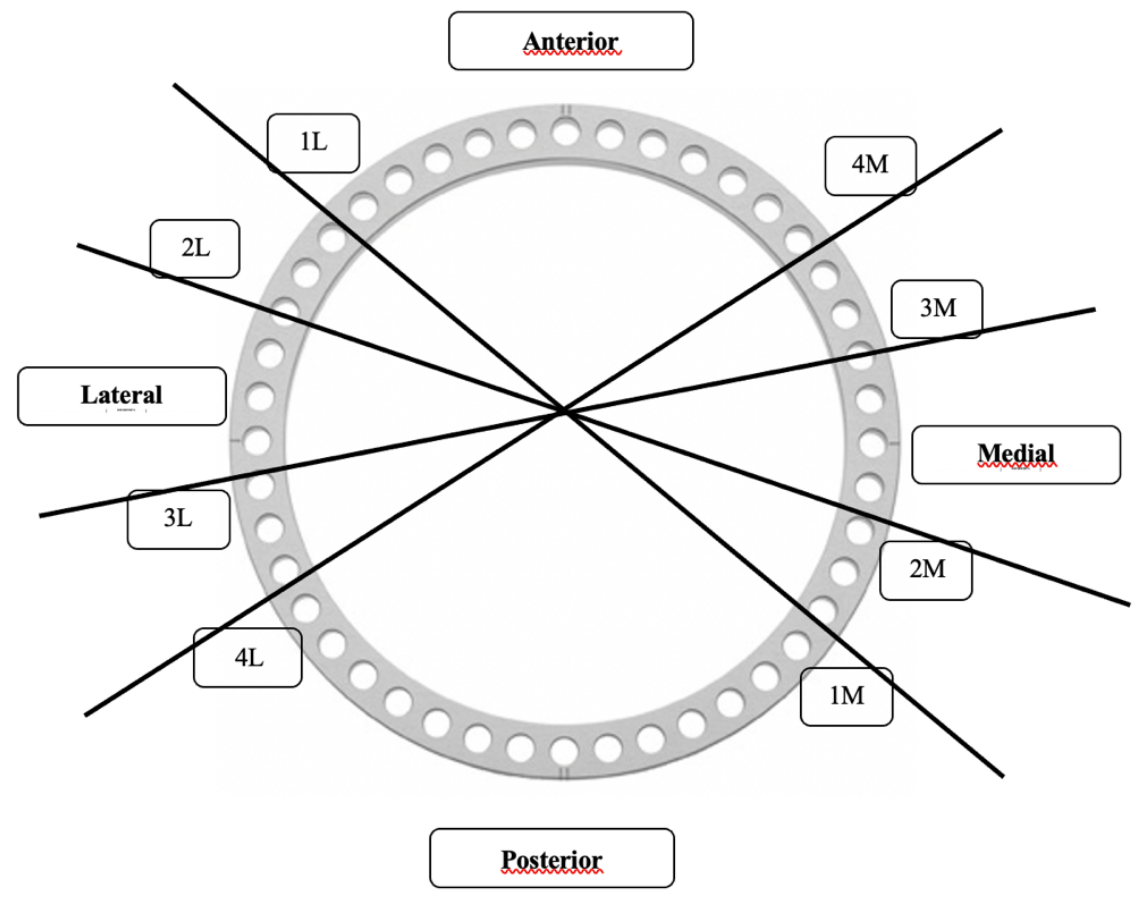


Figure 4S:

Tibia: proximal or distal diaphysis with half pins (standard placement)

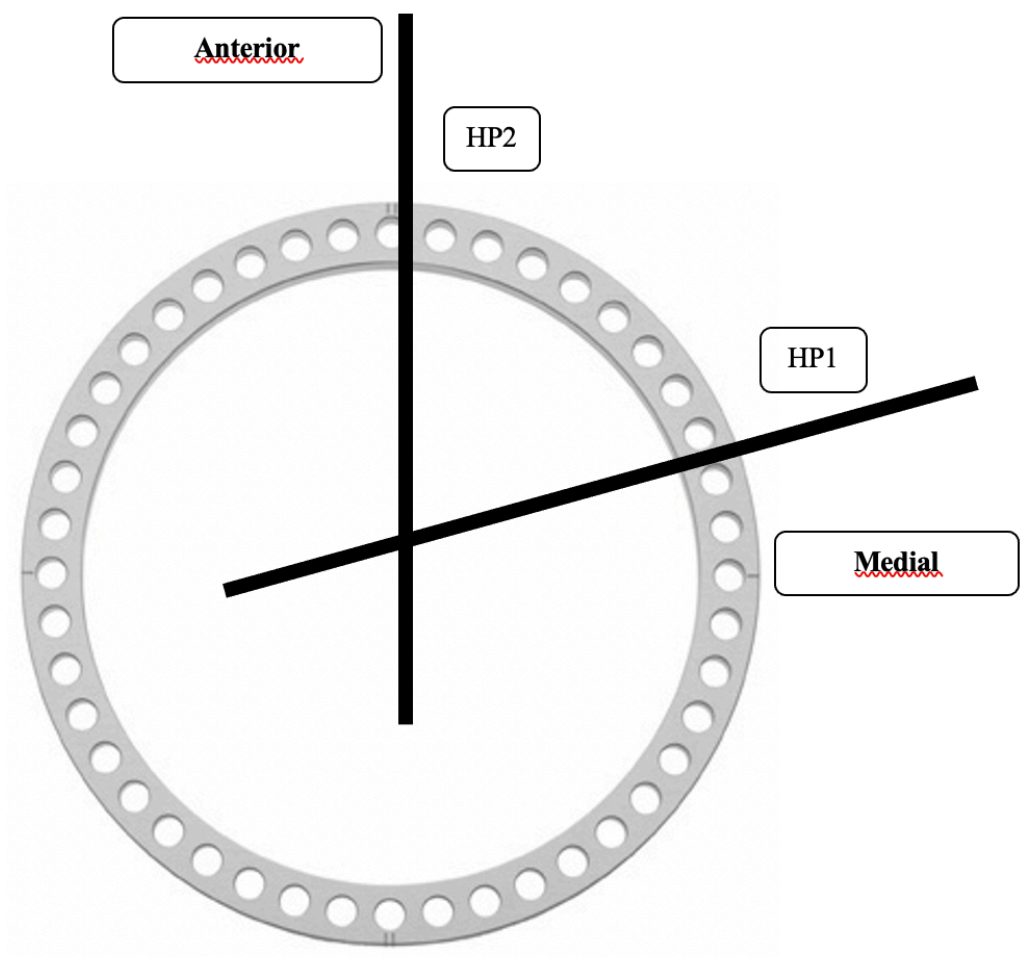

Figure 5S:

100 Footframe: pin sites numbering for standard placement for wires og half pin.

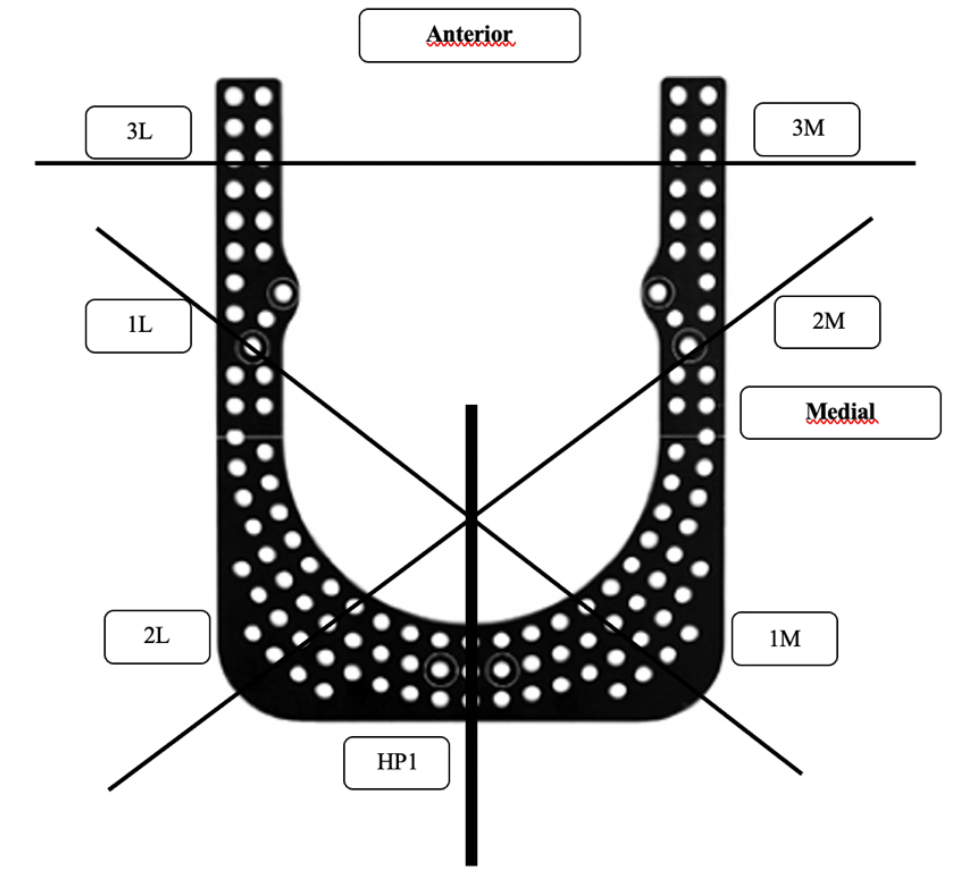

